\title{
An Unusual Complication of Pericarditis
}

\author{
Ashraf Rad ${ }^{1} \quad$ Ulrich Lange ${ }^{2}$ \\ Michael Schmoeckel ${ }^{1}$

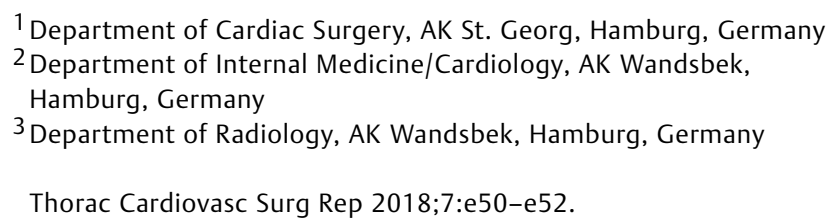

Address for correspondence Michael Schmoeckel, MD, Department of Cardiac Surgery, AK St. Georg, Lohmuehlenstr. 5 Hamburg D-20099, Germany (e-mail: m.schmoeckel@asklepios.com).

\begin{abstract}
Background According to current guidelines, acute pericarditis is an inflammatory pericardial syndrome with or without pericardial effusion.

Keywords

- pericarditis

Case Description We report on a patient who was treated for purulent pericarditis and

- complications developed a fistula from the ascending aorta to the right ventricle (RV), leading to cardiac

- fistula

- left/right shunt decompensation. Operative closure of the aortic and RV perforation solved the problem. Conclusion Bacterial pericarditis may lead to life-threatening complications. Early diagnosis and immediate therapy are essential for a successful outcome.
\end{abstract}

\section{Introduction}

A 47-year-old patient was admitted to the hospital with strong chest pain and suspected transient unconciousness. Clinical examination showed a poor general condition, electrocardiography (ECG) showed a sinus rhythm of 80 per minute, and ST elevation in V3-V5 was diagnosed. Coronary angiography excluded coronary artery disease. Computed tomography (CT) chest and abdomen showed a $2-\mathrm{cm}$ pericardial effusion with increased density, suggesting hemorrhagic or inflammatory origin. There was bilateral pleural effusion and infiltration, and tracheal secretion was positive for Morganella morganii.

Under echocardiographic guidance, a pericardial puncture and drainage was performed, which revealed Streptococcus pyogenes infection. After intervention, transthoracic echocardiography after pericardial puncture showed a normal left ventricular ejection fraction with minimal pericardial effusion $(0.7 \mathrm{~cm})$.

\section{Therapy and Course}

Antibiotic therapy and noninvasive respiratory treatment was initiated with ampicillin/sulbactam and later switched to piperacillin/tazobactam. Noninvasive respiratory treatment was initiated, and the left pleural effusion was drained. After 4 days, the pericardial drainage was removed. Due to

received

August 7, 2018

accepted after revision

October 12, 2018 delirium and respiratory failure, the patient was intubated and ventilated for 3 days. Then he was extubated and transferred to the normal ward. Two weeks after admission, the patient was discharged at his own request against medical advice with the sole therapy of pantoprazole $40 \mathrm{mg}$.

Three weeks later, he was readmitted to the hospital with fever, general weakness with chills, dyspnea, and chest pain. ECG was normal and showed a sinus rhythm of 98 per minute, without any ST segment abnormalities. Chest Xray revealed no signs of infiltration or congestion. Transthoracic echocardiography was essentially normal, and the left atrium (LA) was minimally dilated.

Blood tests showed signs of inflammation: increase of leukocytes and C-reactive protein but normal values for procalcitonin. Therefore no antibiotic therapy was initiated. Creatine kinase (CK) and CK muscle/brain levels were within the normal range.

Eleven days after readmission, the patient was started on corticosteroids (prednisolone $50 \mathrm{mg}$, tapering dose) for recurrent pericarditis and discharged with the request to return to the hospital as soon as his clinical condition would deteriorate. Five days later, the patient was readmitted to the emergency department. He complained of severe dyspnea for the last 3 days (New York Heart Association class III/IV) after one episode of chest pain 3 days ago. Clinical examination (ECG) showed a reduced general condition, normal consciousness, no without fever, and no pain: the patient
License terms Stuttgart · New York

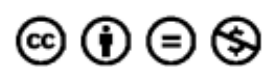


was in sinus rhythm (101 per minute), but now T-wave was negative in V5-V6.

On transthoracic echocardiography, the right atrium and right ventricle (RV) were dilated, whereas the LA and left ventricle (LV) appeared normal. There was a septum shift to the LV. Aortic and mitral valves were without pathological findings except for significant tricuspid valve insufficiency with pulmonary hypertension. Inferior vena cava and liver veins were dilated.

In the distal right ventricular outflow tract, 1.5 to $2 \mathrm{~cm}$ below the pulmonary valve a sealed perforation of around 7 to $9 \mathrm{~mm}$ in diameter was detected with a blood-filled sacculation of $5.5 \times 6.5 \mathrm{~cm}$ on the anterior aspect of the RV within the pericardium ( - Fig. 1). No pericardial effusion or tamponade was seen.

Thoracic CT with contrast radiograph showed a suspected formation of a pseudoaneurysm with a shunt extending from the proximal aortic arch to the RV closely below the PV with a diameter of $5.5 \times 4.5 \times 7.5 \mathrm{~cm}$ in the anterior mediastinum (-Fig. 2). There were signs of pronounced right heart strain and a pericardial effusion with a maximum extent of $1 \mathrm{~cm}$ with increased density.

\section{Operation}

The patient was immediately transferred to the Department of Cardiac Surgery as an emergency case and brought directly into the operating room. Cardiopulmonary bypass was established through femoral arterial and venous cannulation. Following median sternotomy, we encountered a thick-

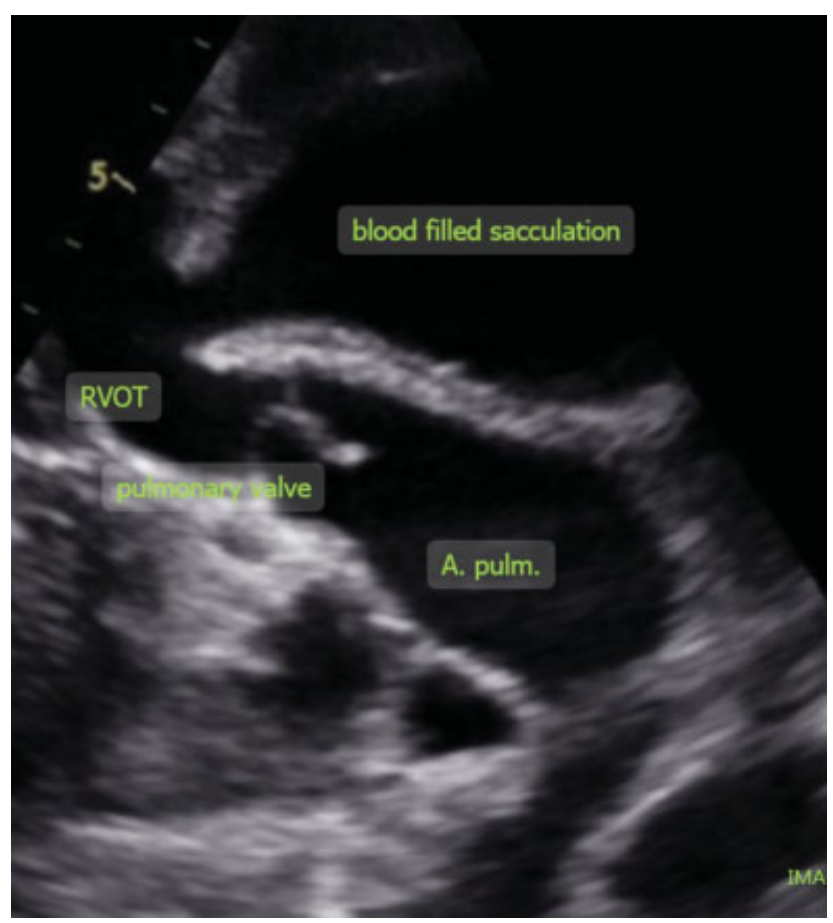

Fig. 1 Parasternal view (third left intercostal space): perforation (7-9 mm in diameter) in the distal right ventricular outflow tract 1.5 to $2 \mathrm{~cm}$ below the pulmonary valve.

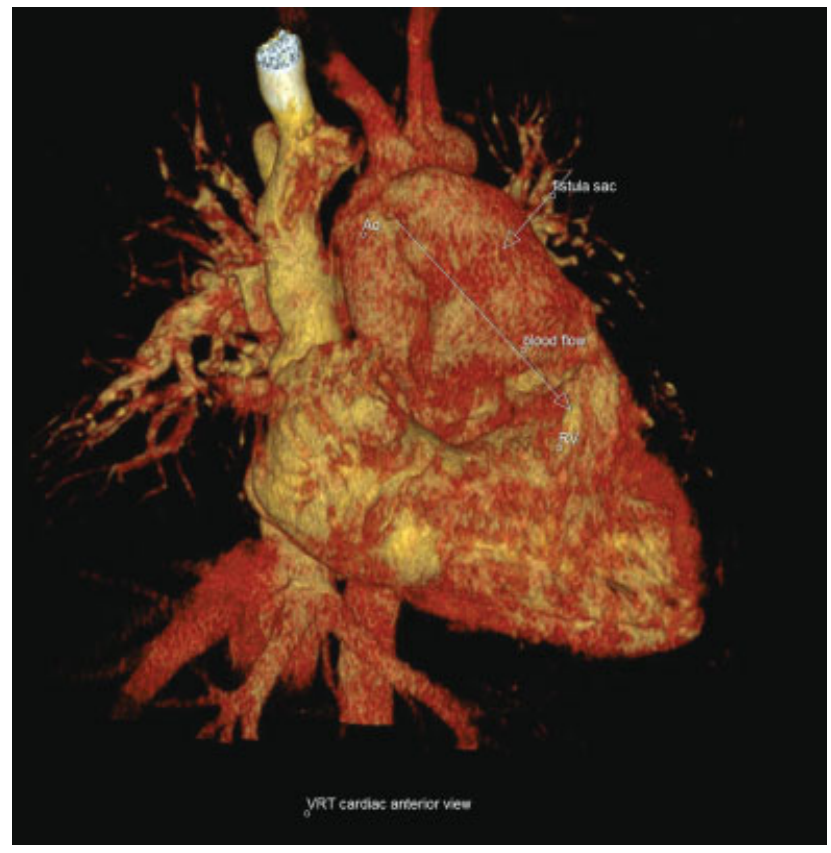

Fig. 2 Three-dimensional reconstruction of aorto-right ventricular shunt.

ened pericardium that was adherent to the epicardium. The RV was massively enlarged; there was diminished contraction in the anterior wall, whereas intraoperative TEE showed normal function of the LV. The superior vena cava was cannulated additionally, and the aortic arch was isolated from adhesions. After aortic cross-clamping distal to the aortic fistula, direct instillation of cold blood cardioplegic solution in both coronary ostia was performed. The fistula was incised anterior to the aorta, and thrombotic material was excised. It appeared that the native aorta had been eroded during pericardial infection, thereby creating a hole of approximately $0.8 \mathrm{~cm}$ in diameter with fibrotic rounded edges. The fistula extended under the epicardium with a cavity up to the middle of the RV partially filled with hematoma. The cavity was connected to the RV by a perforation of approximately $5 \times 4 \mathrm{~cm}$. Thus, we excised necrotic material and closed the perforation with multiple mattress sutures over two felt strips, with subsequent oversewing of the hematoma wall. This was followed by closure of the aortic perforation with a bovine pericardial patch (LeMaitre XenoSure). The operation was terminated as usual, and the patient was extubated the next day.

\section{Postoperative Course}

The patient recovered well. The smears that had been taken during surgery showed growth of Staphylococcus epidermidis. We adjusted the antibiotic regimen according to antibiogram. On the third day after surgery, we transferred the patient to our intermediate care ward. A day later, he was able to stay in a normal ward. On day 11 after surgery, the patient was symptom-free and cardiopulmonary stable and again discharged at his own request with a recommendation to continue intravenous antibiotic therapy. 


\section{Discussion}

Staphylococcus aureus and various streptococci are most common pathogens in purulent pericarditis. ${ }^{1}$ Pneumonia or empyema may be accompanied by bacteremia; patients can develop pericardial infection from hematogenous spread or through microscopic septic emboli into the bronchial arterial circulation, with secondary spread to the pericardium, myocardium, or adjacent mediastinum. ${ }^{2}$ Purulent pericarditis is typically a serious acute illness characterized by high fever, tachycardia, cough, and chest pain. Suspicion of purulent pericarditis is an indication for urgent pericardiocentesis. ${ }^{1}$ The incidence of cardiac tamponade varies from 42 to $77 \%$ in different series; sudden cardiac decompensation can rapidly lead to death. ${ }^{2,3}$ Therefore, purulent pericarditis must be treated aggressively, as death is inevitable if untreated. Intravenous antibiotic therapy should be continued until fever and clinical signs of infection have resolved and until the white blood cell count is normal. In general, this usually consists of a total of approximately 2 to 4 weeks of therapy, depending on the adequacy of drainage and the antimicrobial susceptibility of the infecting bacteria. With comprehensive therapy, $85 \%$ of cases have been reported to survive the episode and have a good long-term outcome. ${ }^{4,5}$ In our case, due to incompliance of the patient, the antibiotic therapy regimen was aborted early and recurrent pericarditis occurred. During anti-inflammatory therapy with steroids, abscess formation led to aortic perforation and eventually formation of a fistula penetrating into the RV.
Substantial left/right shunting developed, leading to right heart decompensation with tricuspid regurgitation and liver congestion. Thus, it can be concluded that in case of high-risk pathogens such as Staphylococcus spp., clinicians should be aware that serious complications such as mycotic aneurysms ${ }^{6}$ or even fistulas creating a hemodynamically relevant shunt may lead to life-threatening situations within a few days.

\section{References}

1 Adler Y, Charron P, Imazio M, et al; ESC Scientific Document Group 2015 ESC Guidelines for the diagnosis and management of pericardial diseases: The Task Force for the Diagnosis and Management of Pericardial Diseases of the European Society of Cardiology (ESC) Endorsed by: The European Association for Cardio-Thoracic Surgery (EACTS). Eur Heart J 2015;36(42):2921-2964

2 Rubin RH, Moellering RC Jr. Clinical, microbiologic and therapeutic aspects of purulent pericarditis. Am J Med 1975;59(01):68-78

3 Boyle JO, Pearce ML, Guze LB. Purulent pericarditis. Review of the literature and report of eleven cases. Medicine (Baltimore) 1961; 40:119

4 Sagristà-Sauleda J, Barrabés JA, Permanyer-Miralda G, Soler-Soler J. Purulent pericarditis: review of a 20-year experience in a general hospital. J Am Coll Cardiol 1993;22(06):1661-1665

5 Imazio M, Brucato A, Mayosi BM, et al. Medical therapy of pericardial diseases: part I: idiopathic and infectious pericarditis. J Cardiovasc Med (Hagerstown) 2010;11(10):712-722

6 Meier D, Kirsch M, Qanadli SD, Muller O, Fishman D, Trana C. Case report of an unusual combination of purulent pericarditis and false aneurysm of the ascending aorta. J Cardiothorac Surg 2018; 13(01):15 